\title{
Movimentação de molares inferiores ancorados em mini-parafusos
}

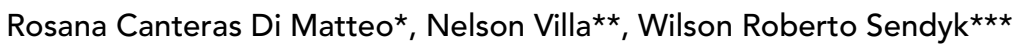

\begin{abstract}
Resumo
Freqüentemente a movimentação ortodôntica exige recursos adicionais de ancoragem. Os mini-parafusos têm-se apresentado como uma possível solução. O propósito deste trabalho foi estabelecer um método para a verticalização de molares inferiores inclinados para mesial, utilizando ancoragem em mini-parafusos colocados na região de linha oblíqüa externa da mandíbula. Foram selecionados três pacientes entre 40 a 48 anos (dois do gênero feminino, um do gênero masculino), com molares inferiores inclinados para mesial e distalmente posicionados às áreas edêntulas. Os pacientes foram tratados ortodonticamente durante um período de 6 a 12 meses, com técnica ortodôntica MD3. Mini-parafusos de titânio foram colocados bilateralmente com anestesia local. Uma incisão sobre a linha oblíqüa externa da mandíbula, medindo aproximadamente $1 \mathrm{~cm}$ foi realizada em cada lado, distalmente aos molares inclinados. Após descolamento muco-periosteal, mini-parafusos foram implantados e foram realizadas suturas deixando suas cabeças exteriorizadas. Uma semana após a remoção das suturas, cargas ortodônticas (entre 150 a 200 gramas/força) foram aplicadas através de forças elásticas. Verificamos que alguma inflamação foi observada ao redor dos mini-parafusos, mas foi controlada com procedimentos de higienização. $O$ procedimento cirúrgico é simples, podendo ser realizado pelo ortodontista; as formas dimensionais dos mini-parafusos são adequadas e estes são de fácil remoção após uso. Concluímos que o uso de mini-parafusos representa uma alternativa efetiva de ancoragem ortodôntica na verticalização de molares inferiores.
\end{abstract}

Palavras-chave: Ancoragem ortodôntica. Implantes. Mini-implantes. Mini-parafusos.

\section{INTRODUÇÃO}

Atualmente, a quantidade de pacientes adultos que procuram tratamento ortodôntico, previamente a tratamentos reabilitadores oclusais é muito significativa.

Em grande número, encontramos mutilações e agenesias dentárias, com inclinações dos dentes posteriores remanescentes, que não permitem uma adequada distribuição das cargas axiais das forças de oclusão, estando associados ainda, a perdas ósseas e problemas periodontais, como bolsas nas faces mesiais destes elementos dentários

* Especialista em Ortodontia e Ortopedia Facial pela Universidade Camilo Castelo Branco. Mestre em Implantodontia pela Universidade de Santo Amaro.

** Professor Associado do Departamento de Histologia pelo Instituto de Ciências Biológicas da Universidade de São Paulo.

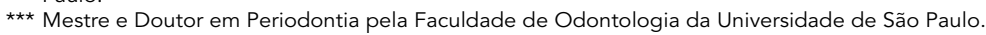


inclinados.

A correção de inclinações insatisfatórias de dentes posteriores inferiores é de alto grau de dificuldade, pois na maioria das vezes, os espaços protéticos situados mesialmente a estes elementos dentários, possuem grandes dimensões, o que dificulta os procedimentos ortodônticos necessários, independentemente da técnica ortodôntica empregada.

Dentro dos procedimentos de correção ortodôntica, sempre nos deparamos com problemas de alterações plásticas da forma dos arcos metálicos de nivelamento dentário, que ocorrem principalmente por forças inadequadas sobre eles durante a alimentação do paciente, e deformações freqüentes destes arcos, acarretam aumento no tempo de tratamento, além de efeitos deletérios nos elementos dentários situados nos extremos do espaço protético e falta de controle na movimentação ortodôntica.

Com o objetivo de melhorar a fisiologia da oclusão dos indivíduos, através do incremento das condições de saúde bucal, a Ortodontia tem se integrado, cada vez mais, às outras especialidades da Odontologia, proporcionando um atendimento multidisciplinar.

Outro aspecto fundamental do tratamento ortodôntico é o controle de ancoragem, ou seja, a resistência ao deslocamento de um ou mais elementos dentários frente a uma força ortodôntica. Todo o aparelho ortodôntico consiste de dois elementos - um elemento ativo e um elemento de resistência. Os elementos ativos, ou elementos do aparelho ortodôntico são as partes referentes ao movimento dentário. Os elementos de resistência proporcionam a ancoragem que possibilita os movimentos dentários e podem ser reforçados pela adição de recursos de ancoragem não dentários como, por exemplo, os implantes.

Dentre os avanços da Ortodontia merece destaque o conceito de ancoragem osseointegrada, uma solução terapêutica capaz de reunir ortodontistas, protesistas, cirurgiões buco-maxilo-faciais e periodontistas em prol da reabilitação da cavidade bucal.

O uso de implantes como ancoragem temporá- ria, facilitando movimentos ortodônticos, tem sido periodicamente relatado na literatura científica nos últimos 40 anos $^{9}$.

O conceito de osseointegração foi estabelecido por Branemark (1965 apud HIGUCHI, SLACK ${ }^{13}$, 1991), mas verificamos a existência de trabalhos anteriores, que vislumbravam este conceito, como o de Gainsforth e Hickey (1945 apud HIGUCHI, SLACK $\left.^{13}, 1991\right)$, que introduziram a idéia de ancoragem por implantes com parafusos de vitálio em mandíbulas de cães.

Branemark et al. ${ }^{4}$ demonstraram que implantes de titânio permaneceram estáveis por períodos superiores a cinco anos, mesmo quando submetidos a grandes cargas. Desde estes estudos iniciais, os implantes de titânio em áreas edêntulas foram amplamente documentados e implantes endósseos mostraram-se práticos e confiáveis adjuntos para o armamento restaurador ${ }^{4}$.

Smith $^{30}$, em um estudo da dinâmica do osso associada ao controle da carga sobre implantes endósseos de óxido de alumínio cobertos com bioglass, sugeriu que era viável incluir o uso de implantes como ancoragem ortodôntica. Afirmou que todos os ossos e raízes dos dentes eram revestidos por alguma forma de tecido conectivo celular, sendo que a aplicação de forças em ossos e dentes era necessariamente mediada por este envelope celular. Com a habilidade de infundir um implante a aplicar forças diretamente ao osso, há uma oportunidade de visualizar as dinâmicas do osso, o que o autor demonstrou não foi o processo de reabsorção e aposição ósseas, mas sim o de estabilidade. As forças foram aparentemente dissipadas, sem causarem uma resposta celular ${ }^{30}$.

Roberts et al. ${ }^{25}$ pesquisaram a aplicação de cargas de 100 gramas durante quatro a oito semanas em pequenos parafusos de titânio colocados em fêmures de coelhos, todos menos um dos implantes permaneceram rígidos. Estes resultados indicaram que implantes de titânio desenvolvem uma interface óssea rígida e que implante sobre carga contínua permanecem estáveis no osso. Estudos 
em animais ${ }^{30,35}$ e em humanos ${ }^{13,24}$ demonstraram que implantes endósseos proporcionam uma modalidade que permite movimento dentário unidirecional sem uma ação recíproca.

Implantes endósseos têm sido usados para promover controle de ancoragem em tratamentos ortodônticos, sem necessidade de cooperação do paciente ${ }^{21,24,29,34}$.

Implantes podem ser úteis na redução do tempo de tratamento, e quando colocados em posições estratégicas a fim de promoverem vetores de forças favoráveis a determinados tipos de movimentos dentários, evitam qualquer tipo de efeito indesejado nos dentes naturais, que previamente receberiam estas forças durante o tratamento ortodôntico.

Kanomi ${ }^{14}$ e Costa et al. ${ }^{8}$ introduziram o uso de mini-implantes para ancoragem ortodôntica. Miniimplantes são pequenos o suficiente para serem colocados em qualquer área do osso alveolar, sendo que este procedimento cirúrgico está ao alcance do ortodontista ou clínico geral, sua cicatrização é rápida e são facilmente removidos após sua utilização. Como mais uma das vantagens, temos que as forças ortodônticas podem ser aplicadas imediatamente após sua implantação ${ }^{22}$, resistindo ao movimento quando submetido à carga desta força ortodôntica ${ }^{3}$.

Trabalhos que demonstram a efetividade da utilização de mini-implantes como ancoragem ortodôntica são fartamente relatados na literatura. Ohmae et al. ${ }^{19}$ avaliaram o potencial desta ancoragem, na intrusão ortodôntica de dentes posteriores inferiores, Park et al. ${ }^{22}$ retraíram dentes anteriores e verticalizaram e intruíram molares mandibulares, Sugawara et al..$^{33}$ corrigiram mordida aberta anterior com a intrusão de molares maxilares, Chung et al. ${ }^{6}$ fizeram retração em massa de dentes anteriores.

O objetivo deste trabalho é propor uma técnica, que utiliza mini-parafusos de osteosíntese como recurso de ancoragem, auxiliando a movimentação de molares inferiores, corrigindo sua axialidade, ou seja, verticalizando-os, para melhor recebimento das cargas oclusais, com controle de ancoragem e minimi- zação de efeitos deletérios dos elementos dentários envolvidos no tratamento ortodôntico.

\section{MATERIAIS E MÉTODO}

A amostra foi coletada aleatoriamente, de acordo com a matrícula dos indivíduos na clínica particular de Ortodontia da autora.

Foram selecionados para este estudo, pacientes de ambos os gêneros (dois do gênero feminino e um do masculino), com idades variando de 40 a 48 anos, com má oclusão de Classe I ou II de Angle, apresentando ausências e agenesias de elementos dentários posteriores inferiores bilateralmente, mas com pelo menos um molar, que poderia ou não apresentar problemas periodontais, situado distalmente à área edêntula, sendo que o mesmo devia apresentar uma inclinação para mesial maior que 20 graus, inclinação esta obtida pelo ângulo formado entre uma reta que une a cúspide disto-vestibular do molar a ser verticalizado com a incisal do incisivo inferior mais posicionado para oclusal e uma reta tangente à oclusal deste molar.

Os pacientes fizeram documentação ortodôntica como pré-requisito para tratamento e foram tratados ortodonticamente com aparelho fixo e técnica ortodôntica MD3 ${ }^{17}$ seguindo todos os passos mecânicos necessários.

A avaliação clínica da presença das linhas oblíqüas externas da mandíbula foi feita através da visualização e palpação digital. A escolha desta área anatômica baseou-se no fato desta estar próxima ao local de interesse, apresentar uma cortical óssea adequada e por não ser passivel de seqüelas irreversíveis.

Os pacientes foram submetidos à tomografia computadorizada de mandíbula com reconstrução tridimensional, enfatizando as regiões dos trígonos retro-molares e linhas oblíqüas externas, a fim de serem observadas as alturas e espessuras ósseas disponíveis e a localização dos canais mandibulares, sendo que este exame complementar, em um dos casos, foi realizado após a montagem dos aparelhos ortodônticos fixos.

Previamente à montagem do aparelho orto- 
dôntico fixo, os pacientes receberam tratamento especializado de raspagem e polimento coronário e radicular, a fim de controlar os problemas periodontais presentes. Receberam ainda, orientação de higienização bucal e tiveram treinamento assistido para reforço do aprendizado.

As cirurgias de colocação dos mini-parafusos foram realizadas na Clínica de Pós-graduação da UNISA.

A medicação pré-operatória profilática utilizada 1 hora antes do procedimento, foi um grama do antibiótico Amoxicilina $500 \mathrm{mg}(\mathrm{lg})$, sendo que nenhum dos pacientes apresentava quadro alérgico a este medicamento.

Utilizou-se como profilaxia intrabucal, o Gluconato de Clorexidina a $0,12 \%$ e extrabucal a clorexidina $2 \%$ solução alcoólica. Realizou-se anestesia local pela técnica pterigomandibular com complementar bucal, utilizando Cloridrato de Mepivacaina a 2\% com adrenalina.

Foi selecionado como implante temporário de carga imediata para ancoragem ortodôntica, um mini-parafuso de osteossitese (bone graft fixation Mincro-Osteomed - Dallas - Texas), com 1.6 mm de diâmetro por 10 a 12 mm de comprimento (Fig. 1). Para a escolha do comprimento dos parafusos considerou-se a espessura muco-gengival de cada caso.

$\mathrm{Na}$ cirurgia propriamente dita, foi realizada uma incisão sobre a linha oblíqüa externa da mandíbula, medindo aproximadamente $1 \mathrm{~cm}$.

Esta incisão iniciou-se no ponto da projeção ortogonal da face distal do molar a ser verticalizado sobre a linha oblíqüa externa na mandíbula indo para trás, sobre a mesma, em direção ao ramo mandibular.

Posteriormente, realizou-se descolamento mucoperiosteal com exposição de parte da linha oblíqüa externa; o mini-parafuso foi implantado no tecido ósseo a uma profundidade de 5 a 6 $\mathrm{mm}$, após o rompimento da cortical óssea através de um broca de 1,3 $\mathrm{mm}$ de diâmetro, utilizando motor com 1600 rotações por minuto, contra-ângulo redutor 1:16 e irrigação central abundante (Fig. 2, 3, 4 e 5).
Realizou-se a sutura, deixando a cabeça do mini-parafuso exteriorizada na cavidade bucal, com a ligadura elástica em posição.

Uma semana após da remoção das suturas, além da mecânica ortodôntica já em andamento, iniciou-se a verticalização dos molares, unindo estes elementos dentários aos mini-parafusos, através de ligaduras elásticas, com forças médias variando de 150 a 200 gramas/força, levando-se em consideração os princípios biomecânicos de direção e quantidade de forças.

A componente vestibular de força nos molares foi neutralizada, pela mecânica ortodôntica fixa.

Sempre que necessário, foram realizadas consultas de orientação de higienização e tratamento profilático necessário.

A cada consulta, a força dos elásticos foi checada através de um tensiômetro e quando necessário, as ligaduras elásticas foram substituídas a fim de que a quantidade de força fosse mantida constante.

Após a verticalização dos molares, um mês antes da moldagem de trabalho para a construção do aparelho ortodôntico de contenção, os mini-parafusos foram removidos em minha clínica particular.

Devido ao tamanho e modo de implantação, os mini-parafusos diferem muito dos implantes convencionais e o fato destes não osseointegrarem-se completamente, facilita sua remoção após uso, como pudemos verificar no momento da remoção dos parafusos na amostra de pacientes utilizada. Estas remoções foram realizadas após anestesia infiltrativa, num procedimento rápido e indolor, sendo que após uma semana os pacientes não apresentavam nenhuma queixa clínica.

\section{DISCUSSÃO}

Restaurar a oclusão estética e funcionalmente em indivíduos com dentes severamente inclinados, exige uma redução significativa da estrutura dentária durante o preparo protético, podendo levar, em alguns casos, à exposição da polpa dentária. Além de 


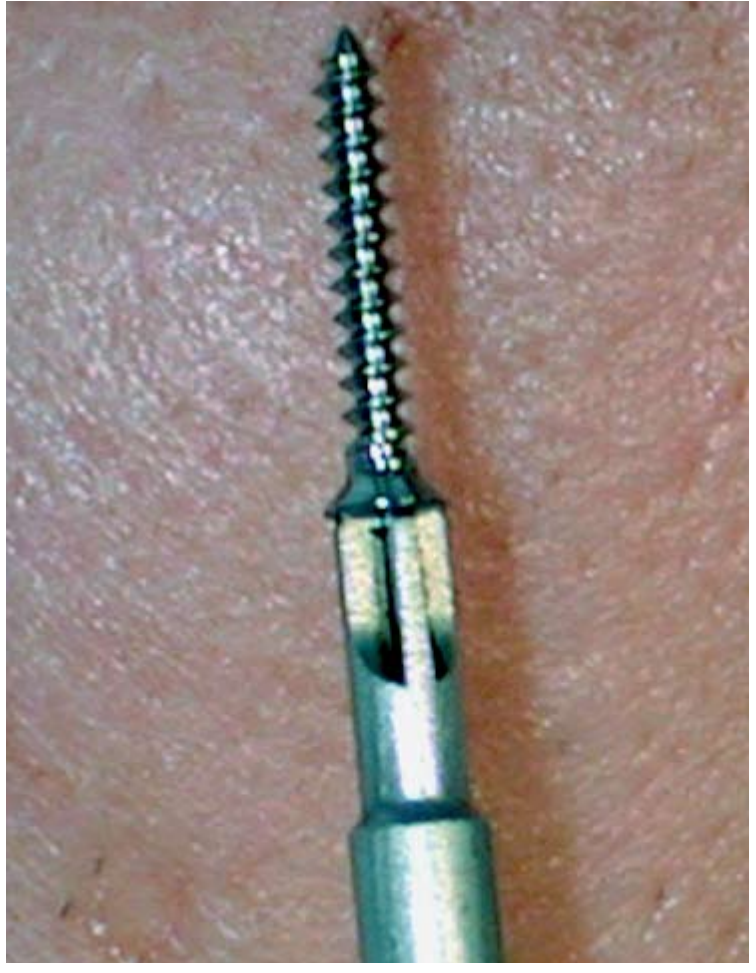

FIGURA 1 - Detalhe do mini-parafuso.

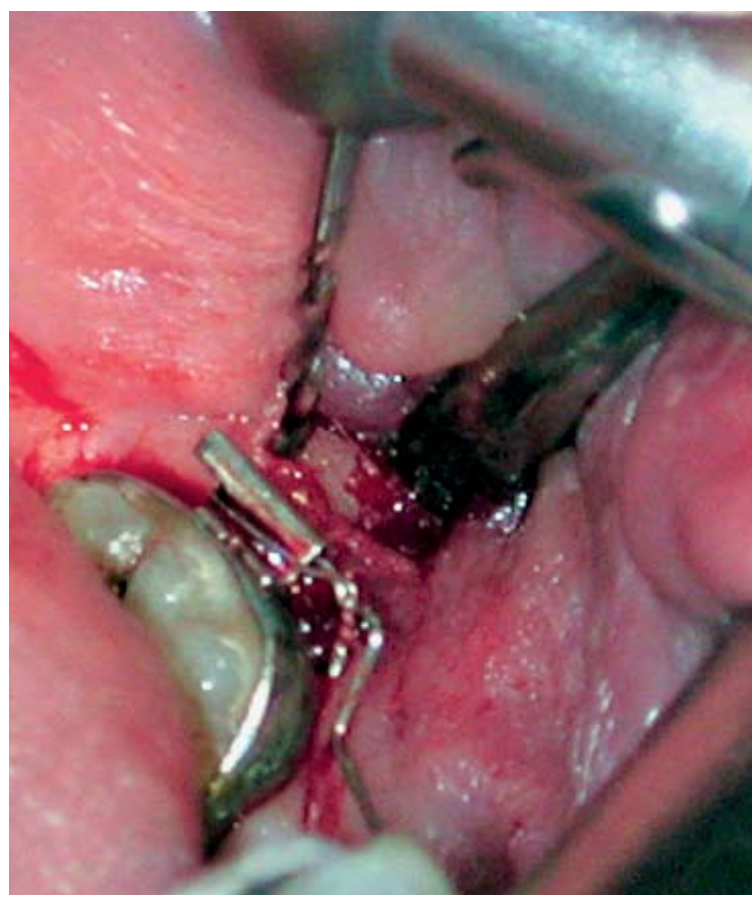

FIGURA 3 - Construção da locação do mini-parafuso.

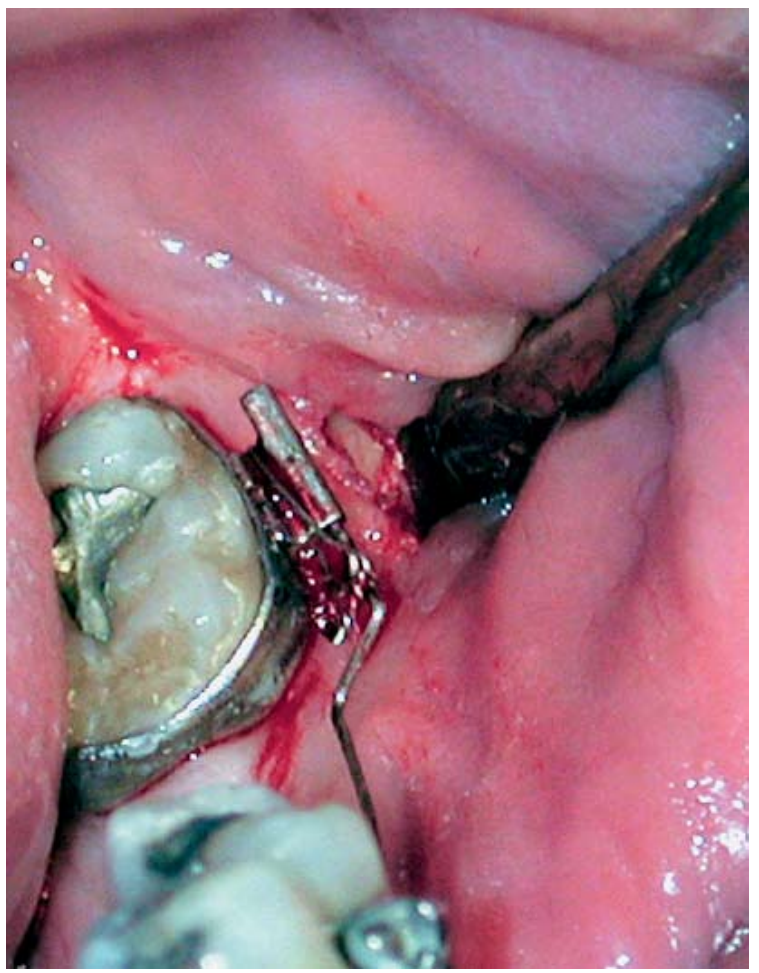

FIGURA 2 - Descolamento muco-periosteal para exposição da linha oblíqüa externa da mandíbula.

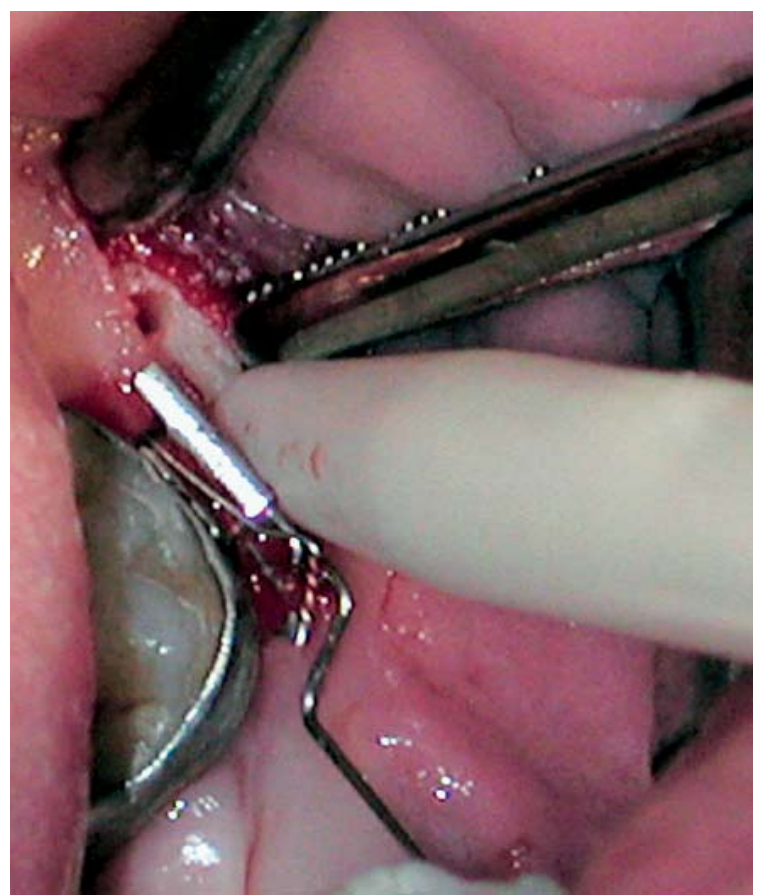

FIGURA 4 - Detalhe do nicho de locação do mini-parafuso com broca 1,3 mm. 


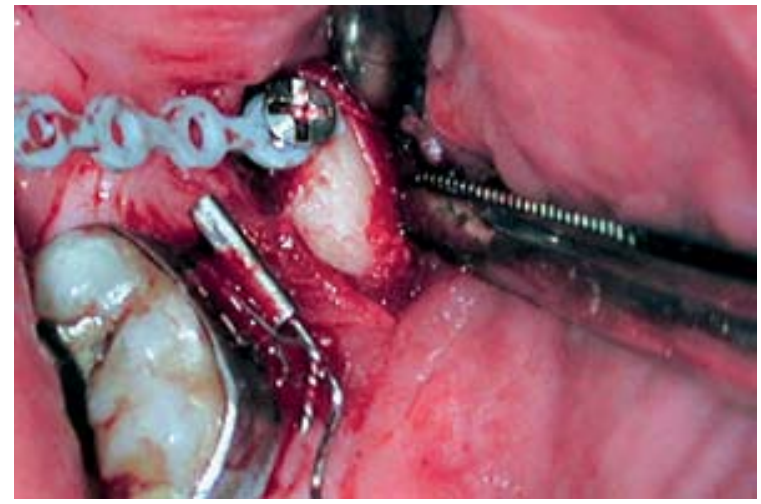

FIGURA 5 - Mini-parafuso com ligadura elástica em posição.

dentes inclinados levarem a problemas periodontais. Nesta situação, verticalizar estes elementos dentários reduz muito os efeitos indesejáveis sobre eles, levando a um resultado final muito mais adequado.

Freqüentemente em reabilitação oclusal, a Ortodontia prévia é necessária por razões funcionais e estéticas.

Sabemos que quando a movimentação ortodôntica exige recursos de ancoragem, freqüentemente são necessários recursos adicionais.

O controle de ancoragem é um desafio difícil e imprevisível na Ortodontia ${ }^{2,8,14,15,36,37}$.

Dentre os avanços da Ortodontia, merece destaque o conceito de ancoragem em implantes, uma solução terapêutica capaz de reunir ortodontistas, protesistas, cirurgiões buco-maxilo-faciais e periodontistas a favor da reabilitação da cavidade bucal ${ }^{10,11,16,18,24,28}$.

Forças aplicadas em implante, não resultam em um processo de reabsorção e aposição óssea como o que ocorre em dentes naturais, mas sim de estabilidade. As forças aparentemente são dissipadas, sem causarem uma resposta celular ${ }^{30}$, mas durante o período de cicatrização os implantes não são rígidos o suficientemente para serem usados como ancoragem ortodôntica, sendo necessário aguardarmos o período de osseointegração ${ }^{35}$.

Para obter uma ancoragem rígida, implantes dentários e parafusos de osteosíntese podem ser utilizados como ancoragem ortodôntica e/ou ortopédica ${ }^{32}$.
Roberts, Marshall e Mozsary ${ }^{24}$ relataram 100\% de rigidez e manutenção na osseointegração em implantes mandibulares após cargas de 300 gramas/força por 13 semanas, mas a literatura demonstra que forças contínuas da ordem de 200 a 600 gramas/forças são compatíveis para a manutenção da osseointegração $0^{40}$.

A osseointegração é mantida pelo estresse mecânico da carga que continuamente estimula a atividade remodeladora do osso, resultando em estabilidade clínica ${ }^{5}$. Posteriormente, este achado foi reforçado por estudos histológicos que avaliavam a osseointegração após carga ${ }^{39,40}$, daí concordarmos com o fato deste procedimento clínico ser sustentável e estável.

O critério de sucesso do uso de implante é determinado quando um ano após o seu uso como ancoragem, nenhum sinal clínico e/ou radiográfico de alteração pode ser observado ${ }^{1}$.

O mínimo estresse do paciente, combinado à máxima ancoragem que o implante proporciona, tornam esta modalidade de tratamento promissora. A quantidade média de forças ortodônticas exercidas sobre estes implantes, a fim de corrigir os mais variados tipos de más posições dentárias, sofre uma variação de 150 a 400 gramas/força 2,11,13,19,38,40.

A literatura relatou a quantidade de força necessária para a movimentação ortodôntica em humanos e em várias outras espécies de animais (ratos, gatos, cães, coelhos, macacos e outros). Verificou-se que a eficiência do movimento ortodôntico em humanos é limitada e a velocidade do movimento não é diretamente proporcional à magnitude da força. A translação ortodôntica é inversamente relacionada à densidade óssea, quanto menor a densidade mais fácil a movimentação dentária ${ }^{26}$.

Considerando a quantidade de carga necessária para a movimentação ortodôntica, foram aplicadas em nossa amostra forças entre 150 e 200 gramas/ força, forças estas mantidas durante todo o movimento de verticalização dos molares inferiores.

Resultado histomorfológicos, a longo prazo, tem demonstrado uma elevação da atividade de remo- 
delamento ósseo tanto em humanos como em outras espécies como coelhos e cães. Na prática clínica, quando os implantes recebem carga ortodôntica, a avaliação da mobilidade e da posição a longo prazo, nos informa sobre sua capacidade de ancoragem ${ }^{5}$.

Acreditamos que o uso de implantes representa um desenvolvimento tecnológico na prática odontológica. $\mathrm{O}$ uso de ancoragem rígida para movimentar dentes é um conceito inovador em Ortodontia. A ancoragem ortodôntica em implantes é um procedimento clínico estável e cientificamente susten$\operatorname{tado}^{7,9,13}$. Deixando a terapia ortodôntica mais rápida e segura, sem efeitos deletérios nas estruturas da cavidade bucal ${ }^{32,34}$, possuindo ainda a vantagem que as forças ortodônticas podem ser aplicadas imediatamente após a implantação ${ }^{3,8,14,22}$.

Sabemos que nos últimos anos, foram adotadas estratégias para reduzirem a carga sobre os dentes de ancoragem a fim de minimizar os efeitos indesejáveis das forças sobre estes elementos dentários. Eventualmente os recursos de ancoragem intrabucais mais utilizados podem apresentar limitações quanto ao seu potencial e os recursos extrabucais podem ser rejeitados pelos pacientes. Daí a importância dos implantes como alternativa valiosa de ancoragem intrabucal.

O uso de implantes é atualmente aceito como uma modalidade de tratamento previsível, mas os implantes convencionais somente podem ser colo-

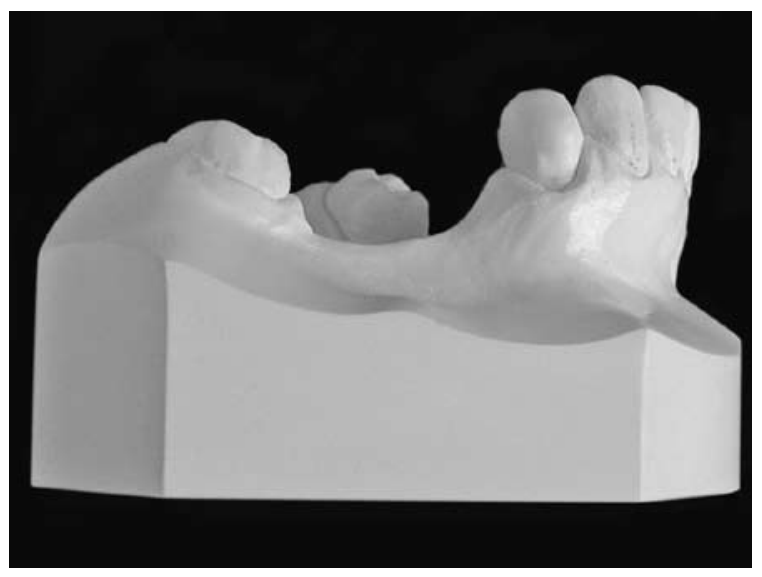

FIGURA 6 - Modelo inferior vista lateral direita pré-verticalização. cados em áreas limitadas na cavidade bucal, como a região retro-molar, rafe mediana da maxila ou áreas edêntulas ${ }^{19,24,25,38}$.

Quando os implantes são colocados em áreas edêntulas, posteriormente poderão ser usados como suporte de próteses na reabilitação oclusal, pois após o uso como ancoragem ortodôntica, mostram-se estáveis a longo prazo como pilares de próteses implanto-suportadas s, $^{9,11,12,16,28,31}$.

Outra limitação seria o sentido da aplicação de força, o implante dentário convencional é colocado no rebordo ósseo alveolar e basal e suas dimensões são grandes, podendo interferir quando da tração ortodôntica horizontal.

Os implantes convencionais podem ser ainda, um problema para o paciente, devido à severidade cirúrgica, desconforto da cicatrização inicial, tempo para a osseointegração e custo.

Quando consideramos os mini-parafusos como recurso de ancoragem ortodôntica, nos deparamos com o fato destes possuírem dimensões muito pequenas, de fácil inserção e localização, incluindo espaços inter-radiculares, a cirurgia é simples, e pouco invasiva, não havendo necessidade de aguardarmos o tempo de osseointegração e de custo menor ${ }^{2}$.

Trabalhos ${ }^{14,15,22,33,37}$ que utilizaram mini-parafusos, verificaram a efetividade da ancoragem, fato este que também comprovamos nos casos por nós tratados, onde verificamos a verticalização dos

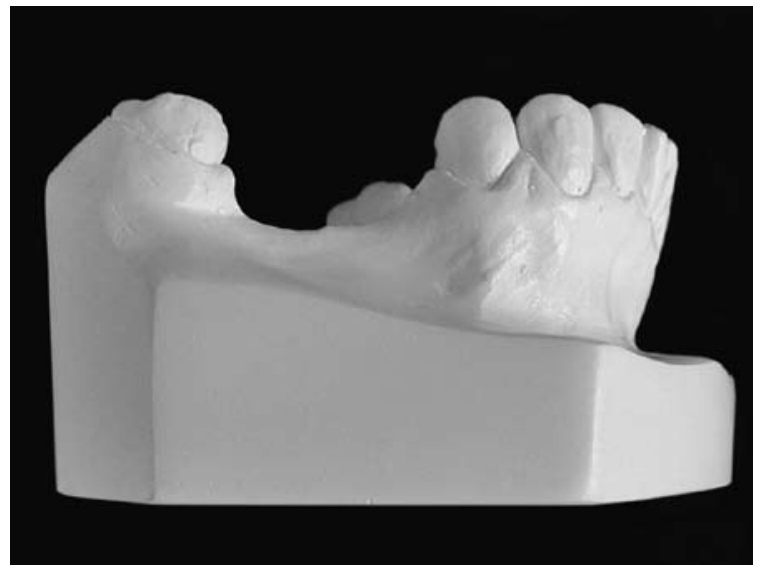

FIGURA 7 - Modelo inferior vista lateral direita pós-verticalização. 
molares inferiores, sem efeitos indesejáveis nos demais elementos dentários (Fig. 6 e 7).

Um outro aspecto discutido na literatura é a presença de mucosite (inflamação) ao redor dos mini-parafusos, havendo um consenso entre os autores que afirmaram ser uma reação inflamatória resultante da falta de controle de higiene do paciente $^{6,7,8,21,24}$, mas que pode ser controlada com a adequação da higiene bucal, associada a bochechos de Gluconato de Clorexidina a 0,12\% $\%^{6,8}$, dado este comprovado em nosso experimento.

Devido ao tamanho e modo de implantação, os mini-parafusos são muito diferentes dos implantes convencionais e o fato destes não se osseointegrarem completamente, facilita sua remoção após o uso $^{2,19,21}$, como pudemos verificar no momento da remoção dos parafusos usados nos pacientes na presente avaliação.

O tipo de mini-parafuso escolhido foi colocado em um tempo cirúrgico, o período de cicatrização foi rápido, o que minimizou o tempo de tratamento, pois a retenção deste mini-parafuso não depende somente da osseointegração, para posteriormente auxiliar na ancoragem para o movimento ortodôntico desejado, mas também do embricamento mecânico entre o mini-parafuso e o osso, que foi ajudado pela inclinação deste, quando na inserção ao osso.

Observamos que pelo fato do mini-parafuso possuir roscas em toda a sua extensão, mesmo na parte que fica em contato com a mucosa gengival, resulta em um fator que dificulta a higienização, pois uma maior quantidade de placa bacteriana fica retida nestas roscas, causando as reações inflamatórias nos tecidos gengivais, as quais foram observadas nos pacientes deste presente estudo.

Em nosso projeto inicial, idealizamos um parafuso com aproximadamente $6 \mathrm{~mm}$ de rosca, que representaria a parte ativa, a qual seria implantada ao osso, o restante de seu comprimento seria liso e ficaria em contato com os tecidos moles, sendo que pelo fato de não possuir roscas, seria menos irritante a estes tecidos e facilitando os procedi- mentos de higiene, minimizando assim, os processos inflamatórios apresentados.

Quanto à cabeça do mini-parafuso, esta poderia ser ligeiramente maior em diâmetro, a fim de proporcionar uma maior retenção para os acessórios ortodônticos necessários, mas não conseguimos encontrar algo semelhante no mercado, então optamos pelo uso de mini-parafusos com dimensões aproximadas às apresentadas na literatura científica.

Se por um lado esta modalidade de ancoragem ortodôntica fica restrita a uma faixa da população, por outro lado nos deparamos com a possibilidade da participação conjunta de diversos profissionais especialistas para um planejamento e um plano de tratamento que devolvam ao paciente a estética e a função esperadas. A abordagem multidisciplinar do paciente é hoje uma realidade.

\section{CONCLUSÕES}

Após avaliação dos nossos resultados de verticalização de molares inferiores ancorados em mini-parafusos podemos concluir que:

1) Não houve restrições tanto no procedimento cirúrgico de implantação como no de remoção dos mini-parafusos.

2) Apresentaram-se como desvantagens no uso dos mini-parafusos:

2.1) A inflamação observada ao redor dos miniparafusos, apesar desta estar diretamente relacionada à higiene de cada paciente.

2.2) O desenho do mini parafuso pode ser otimizado, eliminando-se as roscas que ficam em contato com a mucosa, minimizando assim, a retenção da placa bacteriana.

3) Apresentaram-se como vantagens no uso dos mini-parafusos:

3.1) O baixo custo.

3.2) As dimensões mostraram-se adequadas.

3.3) Os mini-parafusos representam uma alternativa efetiva de ancoragem ortodôntica na verticalização de molares inferiores.

Enviado em: Janeiro de 2004 Revisado e aceito: Abril de 2004 


\title{
Mandibular molar uprighting, using mini-screw as anchorage
}

\begin{abstract}
Tooth movement frequently requires additional anchorage resources. Mini-screws have been used as a possible solution to this matter. The purpose of this study was to establish a method of mandibular molar uprighting, using mini-screw as anchorage, positioned on the mandibular external oblique line, behind and posterior to the tooth. Three pacients with ages between 40 and 48 years old (two females and one male) were selected. The three of them presented mesially tipped molars, positioned posterior to the edentulous areas, on both sides of the mandible. These patients received orthodontic treatment during a period of 6 and 12 months with the MD3 technique. Titanium mini-screws were positioned bilaterally, under local anesthesia. One incision with $1 \mathrm{~cm}$ was done in each side of the mandibular external oblique line, distally to the tipped molars. After that, the mini-screws were positioned and sutures were done, leaving the mini-screws heads exposed. After one week, orthodontic loads (between 150 and 200 grams/force) were applied to the mini-screws through elastics. A certain degree of inflammation around the mini-screws was noticed, but it was controlled by hygienic procedure. This surgery procedure was simple and could be done easily by the orthodontist. The dimensions and shapes of the mini-screws proved to be appropriate and their removal after the orthodontic movement were done without difficulty. The result was time reduction on the molars uprighting, without side effects on the others teeth. The use of mini-screws seems to be a good alternative for an effective orthodontic anchorage on the uprighting mandibular molars.
\end{abstract}

Key words: Orthodontic anchorage. Implant. Mini-implant. Mini-screw.

\section{REFERÊNCIAS}

1. ALBREKTSSON, T. et al. The long-term efficacy of currently used dental implants: a review and proposed criteria of success. Int J Oral Maxillofac Implants, Lombard, v. 1, no. 1, p. 11-25, Summer 1986.

2. BAE, S. M. et al. J. H. Clinical application of micro-implant anchorage. J Clin Orthod, Boulder, v. 36, no. 5, p. 298-302, May 2002.

3. BOUSQUET, F. et al. Use of impacted post for anchorage. J Clin Orthod, Boulder, v. 30, no. 5, p. 261-265, May 1996.

4. BRANEMARK, P. I. et al. A. Intra-osseous anchorage of dental prostheses. I. Experimental studies. Scand J Plast Reconstr Surg, Stockholm, v. 3, no. 2, p. 81-100, 1969.

5. CHEN, J. et al. Mechanical response to functional and therapeutic loading of a retromolar endosseous implant used for orthodontic anchorage to mesially translate mandibular molars. Implant Dent, Baltimore, v. 4, no. 4, p. 246-258, Winter 1995.

6. CHUNG, K. R. et al. The miniplate with tube for skeletal anchorage. J Clin Orthod, Boulder, v. 36, no. 7, p. 407-412, July 2002.

7. CLERCK, H.; GEERINCKX, V.; SICILIANO, S. The zigoma anchorage system. J Clin Orthod, Boulder, v. 36, no. 8, p. 455-459, Aug. 2002

8. COSTA, A.; RAFFAINI, M.; MELSEN, B. Miniscrews as orthodontic anchorage: a preliminary report. Int J Adult Orthodon Orthognath Surg, Chicago, v. 13, no. 3, p. 201-209, 1998.

9. DRAGO, C. J. Use of osseointegrated implants in adult orthodontics treatment: a clinical report. J Prosthet Dent, St. Louis, v. 82 , no. 5, p. 504-509, Nov. 1999

10. FERREIRA, R. I. ; BITTENCOURTE, M. A. V.; CARVALHO, E. M. C. Implantes osteointegrados como ancoragem ortodôntica. Rev Fac. Odonto. Univ Fed Bahia, Salvador, v. 17, p. 84-88; jan./dez. 1998.

11. GOODACRE, C. J. et al. Prosthodontic considerations when using implant for orthodontic anchorage. J Prosthet Dent, St. Louis, v. 77, no. 2, p. 162-170, Feb. 1997.

12. HERRERO, D. B. Implants as anchorage in orthodontics: a clinical case report. J Oral Implantol, Abington, v. 24, no. 1, p. 5-10, 1998.

13. HIGUCHI, K. W.; SLACK, J. M. The use of titanium fixtures for intraoral anchorage to facilitate orthodontic tooth movement. Int J Oral Maxillofac Implants, Lombard, v. 6, no. 3, p. 338-344, Fall 1991.
14. KAMOMI, R. Mini-implant for orthodontic anchorage. J Clin Orthod, Boulder, v. 8, no. 11, p. 763-767, Nov. 1997.

15. LEE, J. S.; PARK, H. S.; KYUNG, H. M. Micro-implant anchorage for lingual treatment of a skeletal Class II malocclusion. J Clin Orthod, Boulder, v. 35, no. 10, p. 643-647, Oct. 2001.

16. LIEBENBERG, W. H. The use of endosseous implant for anchorage during the orthodontic movement of a molar using an upright abutment. J Dent Assoc S Afr, Cape Town, v. 5, no. 3, p. 125-129, Mar. 1996

17. LINO, A. P. Ortodontia corretiva técnica MD3. São Paulo: Artes Médicas, 2001.

18. ÖDMAN, J. et al. Osseointegrated titanium implants: a new approach in orthodontic treatment. Eur J Orthod, London, v. 10, no. 2, p. 98-105, May 1998.

19. OHMAE, M. et al. A clinical and histological evaluation of titanium mini-implants as anchors for orthodontic intrusión in beagle dog. Am J Orthod Dentalfacial Orthop., St. Louis, v. 119, no. 5, p. 189-197, May 2001

20. MARCOTTE, M. R. Biomecânica em ortodontia. 1. ed. São Paulo: Ed. Santos, 1993. cap. 9, p. 147-152.

21. PARK, H. S. et al. Micro implant anchorage for treatment of skeletal Class I bialveolar protrusion. J Clin Orthod, Boulder, v. 35, no. 7, p. 417-422, July 2001.

22. PARK, H. S.; KYUNG, H. M.; SUNG, J. H. A simple method of molar uprighting with micro-implant anchorage. J Clin Orthod Boulder, v. 36, no. 10, p. 592-596, Oct. 2002.

23. REN, Y.; MALTHA, J. C.; KUIJPERS-JAGTMAN, A. M. Angle Orthod, Appleton, v. 73, no. 1, p. 86-92, Feb. 2003

24. ROBERTS, W. E.; MARSHALL, K. J.; MOZSARY, P. G. Rigid endosseous implant utilized as anchorage to protract molars and close an athophic extraction site. Angle Orthod, Appleton, v. 60 , no. 2, p. 135-152, Summer 1990.

25. ROBERTS, W. E.; NELSON, C. L.; GOODAGCRE, C. J. Rigid implant anchorage to close a mandibular first molar extraction site. J Clin Orthod, Boulder, v. 28, no. 12, p. 693-714, Dec. 1994.

26. ROBERTS, W. E.; ARBUCKLE, G. R.; ANALOUI, M. Rate of mesial translation of mandibular molars using implant anchored mechanics. Angle Orthod, Appleton, v. 56, no. 5, p. 331-338, 1996. 
27. SCHWARTZ, H. The case against biomechanics. Angle Orthod, Appleton, v. 37, no. 1, p. 52-57, Jan. 1967.

28. SCHWEIZER, C. M.; SCHLEGEL, K. A.; RUDZKI-JANSON, I. Endosseous dental implants in orthodontic therapy. Int Detal J, London, v. 46, no. 2, p. 61-68, Apr. 1996.

29. SHAPIRO, P. A.; KOKICH, V. G. Uses of implants in orthodontics. Dent Clin North Am, Philadelphia, v. 32, no. 3, p. 539-550, July 1988.

30. SMITH, J. R. Bone dynamics associated with the controlled loading of bioglass-coated aluminum oxide endosteal implants. Am J Orthod, St. Louis, v. 76, no. 6, p. 618-636, Dec. 1979.

31. SORENSON, N. A. Use of maxillary intraosseous implants for Class II elastic anchorage. Angle Orthod, New York, v. 65, no. 3, p. 169-173, 1995.

32. SOUTHARD, T. E. et al. Intrusion anchorage potencial of teeth versus rigid endosseous implants: A clinical and radiographic evaluation. Am J Orthod Dentofacial Orthop, St Louis, v. 107, no. 2, p. 115-120, Aug. 1995

33. SUGAWARA, J. et al. Treatment and posttreatment dentoalveolar changes following intrusion of mandibular molars with application of a skeletal anchorage system (SAS) for open bite correction. Int J Adult Orthodon Orthognath Surg, Chicago, v. 17, no. 4, p. 243-253, 2002.
34. TRISI, P.; REBAUDI, A. Progressive bone adaptation of titanium implants during and after orthodontic load in humans. Int J Periodontics Restorative Dent, Chicago, v. 22, no. 1, p. 31-43, Feb. 2002

35. TURLEY, P. K.; et al. Orthodontics force application to titanium endosseus implants. Angle Orthod, Appleton, v. 58, no. 2, p. 151-162, Apr. 1988.

36. TURPIN, D. L. Orthodontists needed on the implant team. Angle Orthod, Appleton, v. 65, no. 3, p. 163, 1995.

37. UMEMORI, M. et al. Skeletal anchorage system for open-bite correction. Am J Orthod Dentofacial Orthop, St. Louis, v. 115, no. 2, p. 166-175, Feb. 1999.

38. VALERÓN, J. F.; VELÁSQUEZ, J. F. Implants in the orthodontic and prosthetic rehabilitation of an adult patient: a case report. Int J Oral Maxillofac Implants, Lombard, v. 11, no. 4, p. 534-538, July/Aug. 1996.

39. WEHRBEIN, H.; GLATZMAIER, J.; YILDIRIM, M. Orthodontic anchorage capacity of short titanium screw implants in the maxilla. An experimental study in dog. Clin Oral Implants Res, Copenhagen, v. 8, no. 2, p. 131-141, Apr. 1997.

40. WEHRBEIN, H. et al. Bone-to-implant contact orthodontic implants in humans subjected to horizontal loading. Clin Oral Implants Res, Copenhagen, v. 9, no. 5, p. 348-353, Oct. 1998.
Endereço para correspondência

Rosana Canteras Di Matteo

Rua Catequese, 255 sala 13

CEP: 09090-4000 - Santo André/SP

E-mail: rodimatteo@osite.com.br 